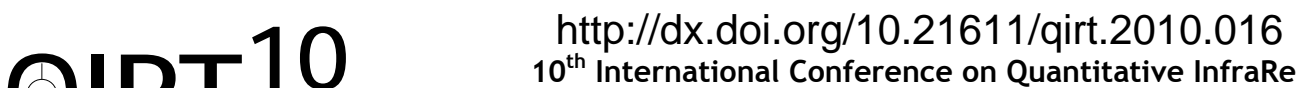

\section{Analysis of infrared images in integrated-circuit techniques by mathematical filtering}

\section{by I. Benkö Prof.Dr.techn.}

Fac.of Mech.Engineering, Budapest University of Technology and Economics, Budapest, Hungary, H-1112 Budapest, Cirmos u.1., Hungary, Fax/Phone:36-1-310-0999,

ibenko@freestart.hu

Keywords: mathematical filtering, infrared images, integrated circuits, computer processing

\section{Abstract}

Computer processing of the infrared (IR) images speeds up considerably the evolution of the measurements results. Processing of digitized IR images may be carried out by a variety of approaches and methods. On the whole, it may be said that it is expedient to tailor the strategy and the method of evaluation to the phenomenon under scrutiny. This, in some cases, calls for both heat engineering experience and technical intuition. Image filtering is a special and not commonly used method of IR image analysis. The present author has no information about publications on the mathematical filtering of IR images. The first paper on that topic by the present author was published in 1997. The mathematical IR image filtering in general is suitable for detecting some thermal faults which are difficult to identify otherwise. Image filtering is based on the adequate transformation of the temperature field, i.e. the modification of the temperature values in the pixels of the image.

\section{Introduction}

This paper covers only the data obtained through the computer processing of infrared (IR) thermograms which had to be evaluated because of the IR images gained from various measurements and the nature of the objectives [1,2]. The methods of filtering can be grouped as follows: smoothing filters, sharpening filters, band filters and gradient filters. It is important to emphasize that, after the filters are applied, the colours and temperatures indicated in the resultant image have no relation to the actual temperatures but to the physical essence of the filtering method selected. The illustrations for IR image analysisare taken from the electronics industry (see figures $1 \mathrm{a}$ and $1 \mathrm{~b}$ ) [3].

\section{Generalities}

The principal objective of image enhancement techniques is to process an image so that results should be more suitable for a specific application than the original image. In the IR thermogrammetry the goal of the image processing is to obatain a new IR image showing some thermal faults or singularities in the temperature field. It is to be noted that the image enhancement techniques are very much problem oriented.

Generally, image enhancement methods are based or either spatial or frequency domain techniques. The spatial domain refers to the image plane itself, and approaches in this category are based on direct manipulation of pixels in an image. Frequency domain processing techniques are based on modifying the Fourier transform of an image (see Section 6).

\section{Spatial domain methodes of image filtering}

The term spatial domain refers to the aggregate of pixels composing an image, and spatial domain methods are procedures that operate directly on these pixels [4]. Image processing functions in the spatial domain may be expressed as

$$
g(x, y)=T[f(x, y)]
$$


where $f(x, y)$ is the input image, $g(x, y)$ is the processed image and $T$ is an operator on $f$, defined over some neighbourhood of $(x, y)$.principal

One of the principal approaches in equation (1) is based on the use of so-called masks (also referred to as templates, windows or filters). Basically, a mask is a small two-dimensional array, in which the values of the coefficients determine the nature of the process, such as sharpening. Enhancement techniques based on this type of approach often are referred to as mask processing or filtering.

\section{Smoothing filters}

Smoothing filters are used for blurring and for noise reduction. Blurring is used in preprocessing steps, such as removal of small details from an image prior to (large) object extraction, and bridging of very small gaps in line or curves. Noise reduction can be accomplished by blurring with a linear filter and also by non-linear filtering.

\subsection{Neighbouring filter}

In this filtering function the value of the pixel is calculated by averaging the values of the neighbouring pixels. Either four or eight neighbouring pixels can be included. For four pixels the equation $g(x, y)$ defined as follows:

$$
g(x, y)=1 / 4 \sum(n, m) f(n, m) .
$$

In the above equation $g(x, y)$ is the modified pixel and $f(n, m)$ is the neighbouring pixels.

\section{Sharpening filters}

The principal objective of sharpening is to highlight fine details in an image or to enhance detail that has been blurred, either in error or as a natural effect of a particular method of image acquisition. Uses of image sharpening vary and include applications ranging from electronic printing and medical imaging to industrial inspection and detection of some thermal faults in a printed circuit in electronic technology.

\subsection{Derivative filters}

Averaging of pixels over a region tends to blur detail in an image. As averaging is analogous to integration, differentiation can be expected to have opposit effect and thus to sharpen an image. The most common method of differentiation in image-processing applications is the gradient.

\subsubsection{Gradient filter}

The gradient filter assesses a pixel with regard to the numerical difference to its neighbourhood pixels. Either of two different gradient equations can be selected:

(a) common (or normal) gradient equation and

(b) the so-called Roberts gradient equation.

They differ in how the the neighbouring pixels are viewed.

The normal gradient. The gradient (difference) equation is defined as follows:

$$
g(x, y)=[f(x, y)-f(x+1, y)]+[f(x, y)-f(x, y+1)]
$$

The result $g(x, y)$ replaces $f(x, y)$.

The Roberts gradient. The gradient equation is defined as follows: 


$$
g(x, y)=[f(x, y)-f(x+1, y+1)]+[f(x+1, y)-f(x, y+1)]
$$

The result $g(x, y)$ replaces $f(x, y)$.

In both gradient filter functions the calculated value of the pixel can be modified by further parameter options.

\section{Matrix filter}

Within the function, three filter subfunctions are available:

(a) low pass,

(b) high pass and

(c) user.

The matrix filter works according to the following mathematical operation. The pixel value of a $3 \times 3$ matrix is replaced by the relation:

$$
g\left(m_{1}, m_{2}\right)=\sum_{n 1} \sum_{n 2} \boldsymbol{F}\left(n_{1}, n_{2}\right) \times \boldsymbol{H}\left(m_{1}-n_{1}-1, m_{2}-n_{2}+1\right)
$$

where $\boldsymbol{F}$ and $\boldsymbol{H}$ represent the image matrix and the convolution matrix respectively.

\subsection{Sobel filter}

The Sobel filter is a special matrix filter which is particularly suitable for recognizing countours. The results are similar to those achieved with the gradient filter (see Section 5.1.1.).

In the Sobel filter the gradient equation is the follows:

$$
g=\mathbf{w}_{1} \mathbf{x}+\mathbf{w}_{1}{ }^{\mathrm{t}} \mathbf{x}
$$

where $\mathbf{w}_{1}$ is the $3 \times 3$ weight matrix that the user has to define, and $\mathbf{w}_{1}{ }^{\mathbf{t}}$ is the weight matrix transposed from $\mathbf{w}_{\mathbf{1}}$ and $\mathbf{x}$ is the $3 \times 3$ image section matrix. For pixel modification the centre pixel of the image section matrix $\mathbf{x}$ is replaced. This is always taken from the original image.

\section{Analysis of thermal singularities of an integrated circuit}

The applicability (advantages and limitation) of IR images and their scientific value are determined in the technical practice by the following main features:

(a) the type of the technical phenomenon investigated,

(b) consideration of rules of IR optics during the IR image taking and

(c) proper selection of ambient parameters.

Under optimum conditions, the IR image will contain important information on the thermal character of the temperature field of the examined object or phenomenon. As a consequence, the evaluation of IR images, the thermal physical characteristics and the interpretation will contribute to the scientific value of IR images.

In the actual practice of IR image analysis the following general methods may be chosen, while their relative advantages and disadvantages must be decided in the light of the test being done. The first is the traditional phenomenological analysis, e.g.determination of the temperature at specified points of the (e.g. the centre point of the cross hairs) (see Fig. 3a), a comparison between temperature distribution along the horizontal, vertical or optional lines (see Fig.3a), and relief representation of the temperature field (see Fig.3c). Two new methods serve for mathematical evaluation of temperature fields:

(a) histographic analysis, i.e. the application of the distribution curve of temperature histogram for process monitoring (see Fig. 3b) [5] and

(b) mathematical filtering of IR images to reveal the sites of highest temperature or of the largest temperature alteration (see Fig.2). 
In the section several techniques are presented for the enhancement and evaluation of IR images. The illustration are taken from the field of integrated-circuit techniques(figure 1).An example for the filtering of the component side is shown in Fig. 2b (Sobel) and the applied Roberts gradient in Fig.2c, where the thermal singularities can be seen. Therefore the recommendable process of mathematical filtering applying to the original IR images in similar technical cases is as follows. The firs step is the Sobel filter which is a sharpening step, equalizing the smaller temperature differences so that the contours of the high-temperature field can bee achieved (Fig.2b). The second step is subsequent filtering of figure2b by the Roberts gradient. The Roberts gradient filtering ensures that all the pixels have a gradient value larger than a threshold and the other filtering provides a background level value. Therefore figure 2 has a threshold of 80 and a background of 15 and shows the 'hot field' of the component side of the integrated circuit.

A common evaluation for analysis of the chip side (Fig.1b) is is presented in figure 3. For comparison of 'clasical' and filtering evaluation of IR images, figure 3 presents some possibilities for chip side of the integrated circuit (Fig.1b). Figure 3a shows the temperature distribution along lines created horizontally and vertically, similarly to Fig.2a. Figure $3 b$ is the histographic processed result of Fig.3a, representing the more significant characteristics of the histogram in the specified area in Fig.3b: the highest (maximum $65.4^{\circ} \mathrm{C}$ ), the lowest (minimum $28.6^{\circ} \mathrm{C}$ ) and the middle (average $37.7^{\circ} \mathrm{C}$ ) temperature,etc. Figure $3 \mathrm{c}$ is a three-dimensional relief display of Fig.3a.

When the spots with the highest temperatures on both the chip and the component sides are known, the faulty or improperly sized elements can be replaced. Thus unexpected later failures due to the high local thermal load can be prevented.

\section{Conclusions}

IR image filtering is a practical, quick and normalizable evaluation method with the aim of analysing the temperature field initially. The topic presented in this paper is representative of techniques that are commonly used in practice for digital IR image enhancement. However, this area of image processing is basically problem oriented, and a dynamic field in the literature, too. For this reason, the methods included in this paper have to select both the topic and the goal of thermal analysis.

\section{REFERENCES}

[1] Benkő, I. Applications of infrared thermogrammetry in thermal engineering. In Proceeding of the Conference on Quantitative Infrared Thermography (QIRT 92), Eurotherm Séries 27, pp.343-349 (Edition Européennes Thermique et Industrie), Paris (1992).

[2] Benkö, I. Infra-red picture analysis in thermal engineering. In Proceedings of the Tenth International Conference on Thermal Engineering and Thermogrammetry (Ed. I.Benkő),pp.46- 52 (MATE TE and TGM),Budapest(1997).

[3] Benkö, I. Applications of infrared filtering in electronic technology. In Book of Abstracts of Quantitative Infrared Thermography (QIRT 98) (Ed. B. Wiecek) pp.86-87 (PKOptoSEP), Lodz(1998).

[4] Gonzales, R.G. and Woods, R.E. Digital Image Processing (Addison-Wesley), Wokingham(1977).

[5] Benkö, I. ; Köteles, G.J. and Németh, G. New infrared histographic investigation of the effect of beta-irradiation in medical field. In Proceedings of the Conference on Medical Infrared Thermography (MIRT 98) (Eds. D. Balageas, G. Busse, C. M. Carlomagno and B.Wiecek) pp.40-45 (PKOptoSEP), Lodz(1998). 


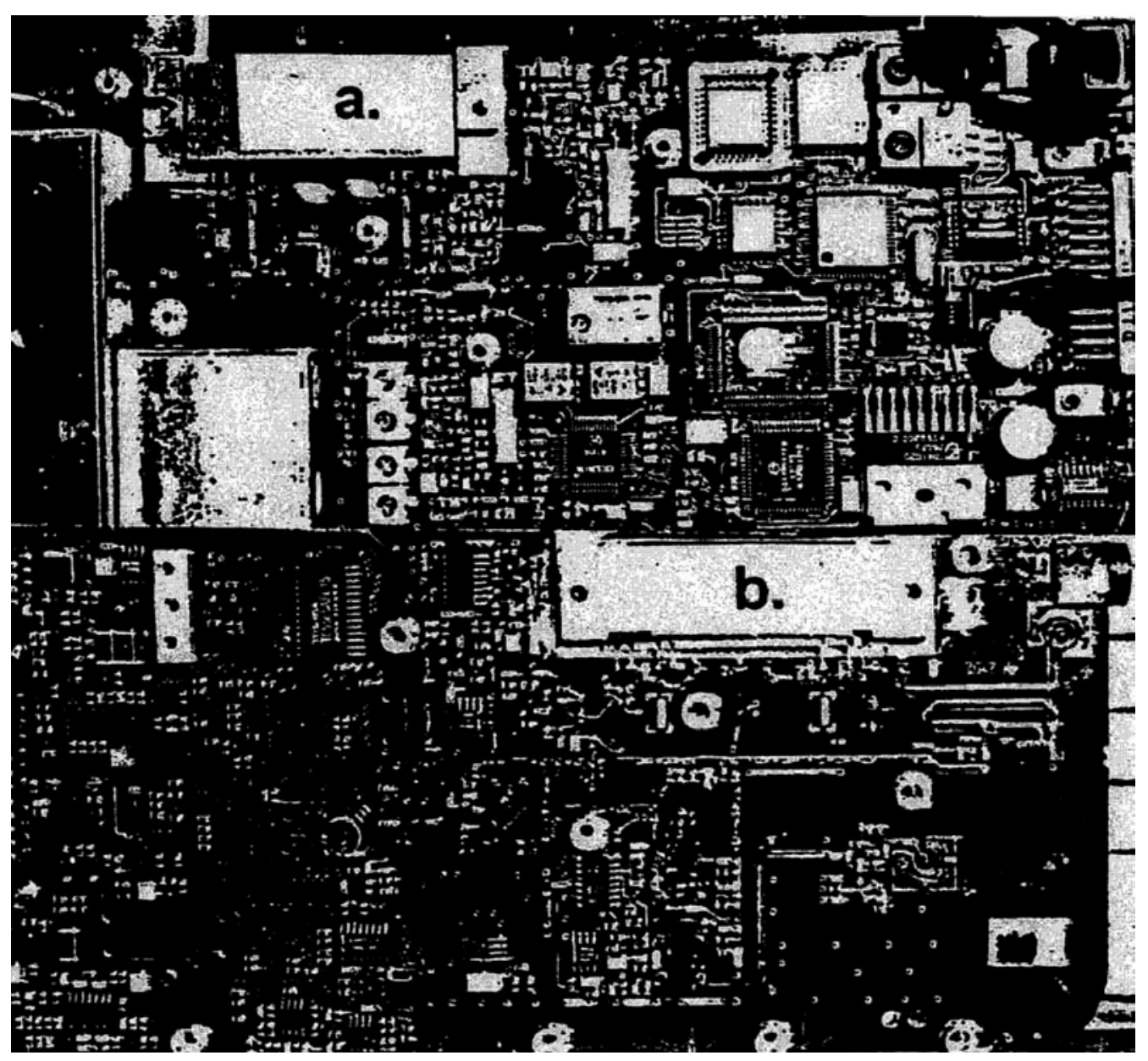

Fig.1, (a) The component side and (b) the chip side of an integrated circuit 


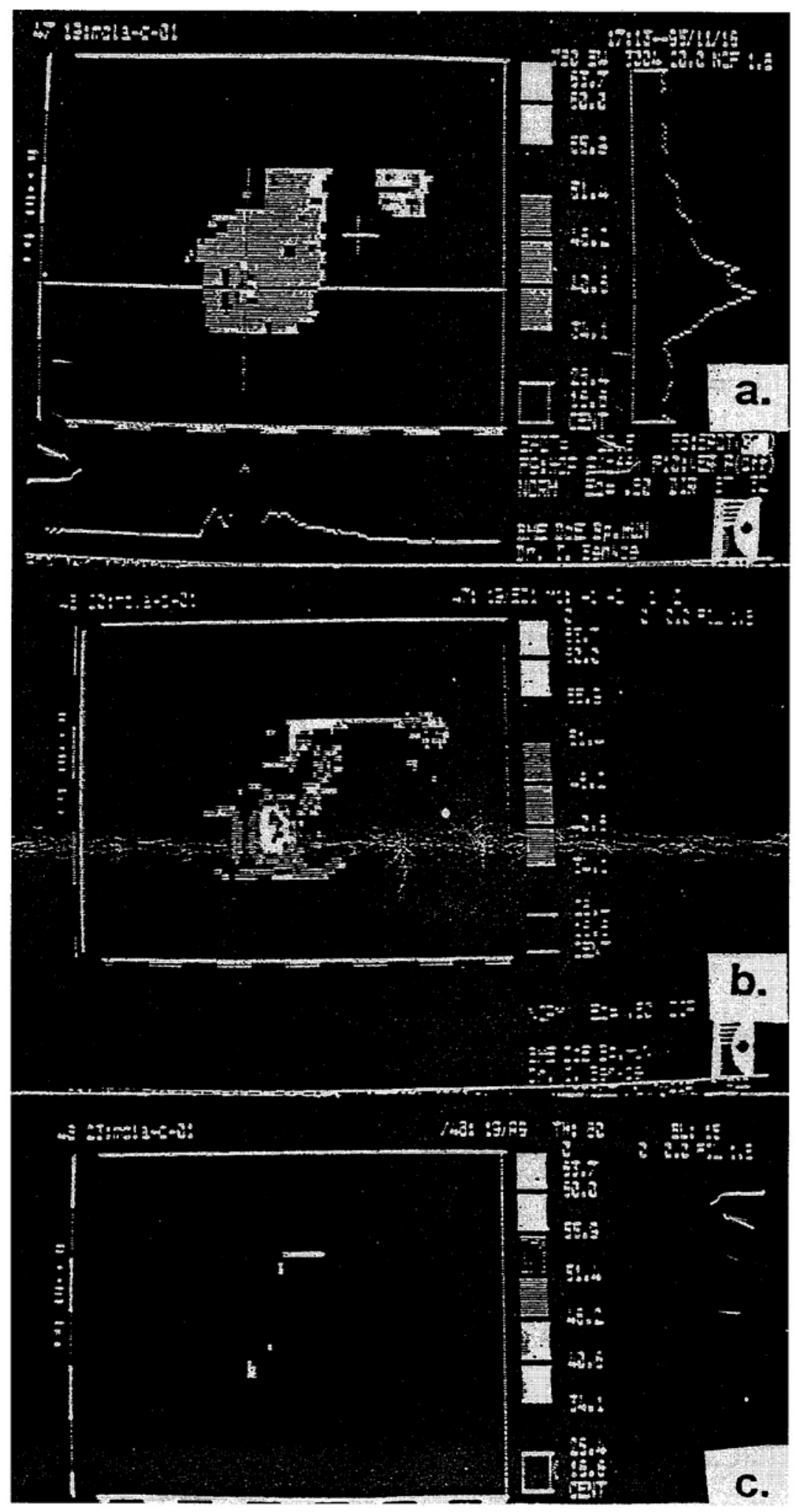

Fig. 2. Series of IR images concerning the filtering of Fig. 1a: (a) original IR image; (b) first step (the Sobel filter) of filtering; (c) second step (the Roberts gradient) of filtering 


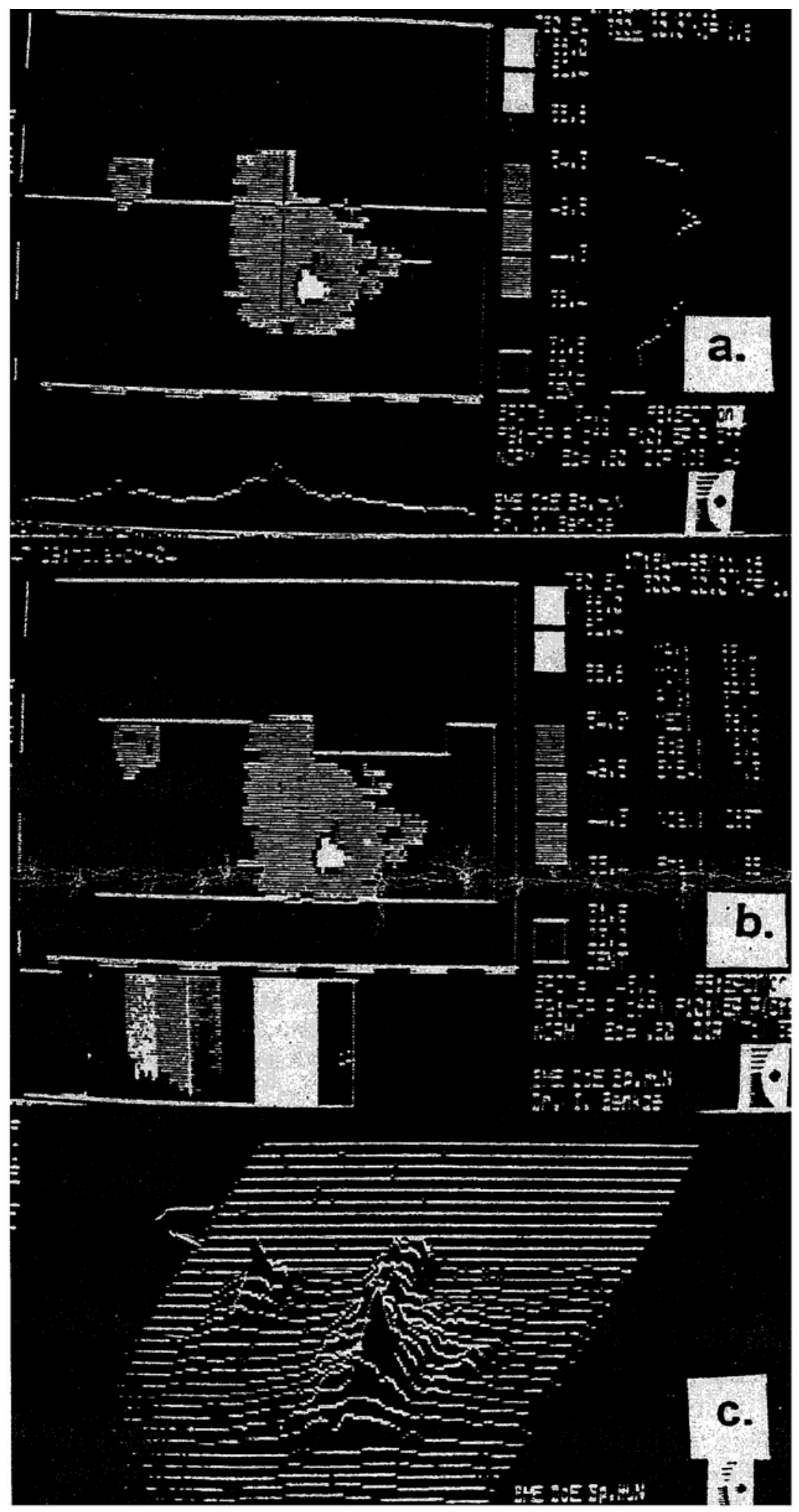

Fig. 3. Common evaluation of IR images on the chip side; (a) line thermogram; (b) histogram; (c) relief 